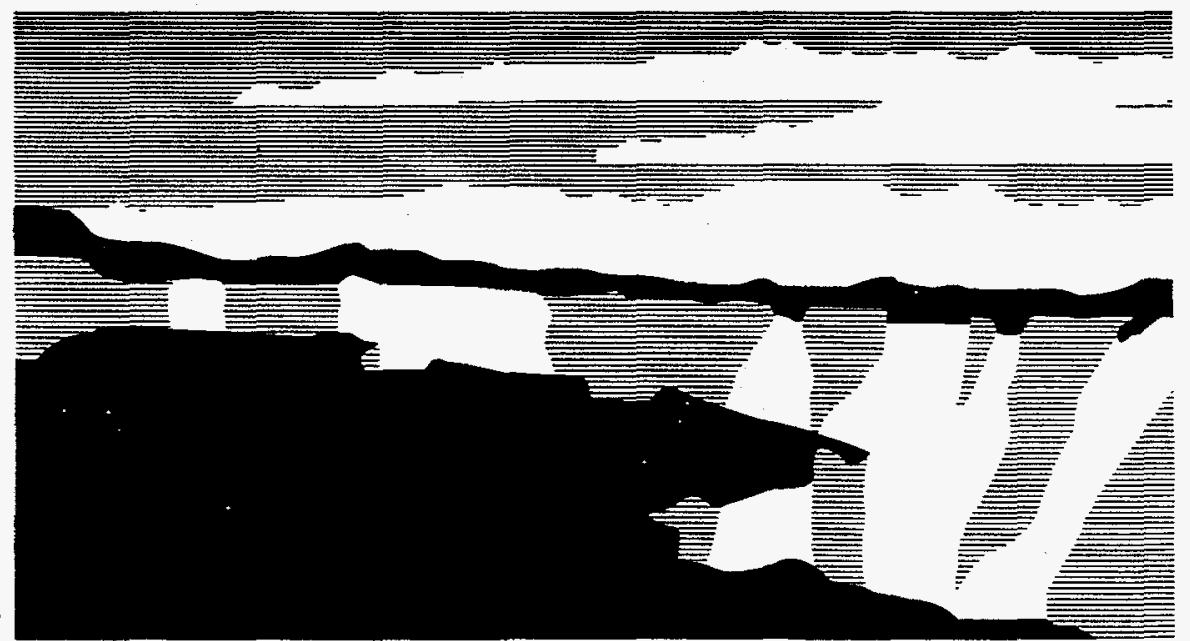

Los Alamos National Laboratory, an affirmative action/equal opportunity employer, is operated by the University of California for the U.S. Department of Energy under contract W-7405-ENG-36. By acceptance of this article, the publisher recognizes that the U.S. Government retains a nonexclusive, royalty-free license to publish or reproduce the published form of this contribution, or to allow others to do so, for U.S. Govemment purposes. The Los Alamos National Laboratory requests that the publisher identify this article as work performed under the auspices of the U.S. Department of Energy.

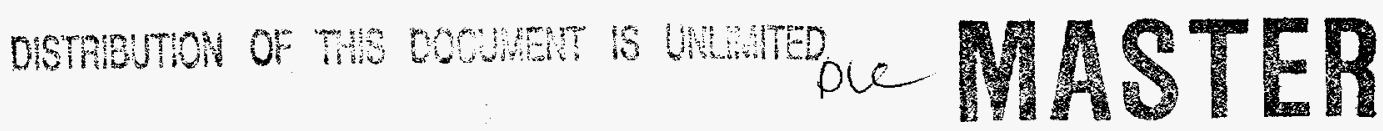




\title{
Linear Accelerator for Tritium Production
}

\author{
R. W. Garnett, J. H. Billen, K. C. D. Chan, R. Genzlinger*, \\ E. R. Gray, S. Nath, B. Rusnak, D. L. Schrage, J. E. Stovall, \\ H. Takeda, R. Wood, T. P. Wangler, and L. M. Young
}

\author{
Accelerator Operations and Technology Division, \\ Los Alamos National Laboratory, \\ Los Alamos, New Mexico 87545
}

\begin{abstract}
For many years now, Los Alamos National Laboratory has been working to develop a conceptual design of a facility for accelerator production of tritium (APT) [1]. The APT accelerator will produce high energy protons which will bombard a heavy metal target, resulting in the production of large numbers of spallation neutrons. These neutrons will be captured by a low- $Z$ target to produce tritium. This paper describes the latest design of a room-temperature, $1.0 \mathrm{GeV}, 100 \mathrm{~mA}$, cw proton accelerator for tritium production. The potential advantages of using superconducting cavities in the highenergy section of the linac are also discussed and a comparison is made with the baseline room-temperature accelerator.
\end{abstract}

\section{DESIGN PHILOSOPHY}

The main design objectives for the APT accelerator are to provide high beam transmission and low beam loss during all phases of linac operation. Advances of the past decade have made it possible to design a machine of this type by providing the framework required for emittance and beam halo control of high-current proton beams. The use of high-frequency accelerating structures reduces the amount of charge per bunch which minimizes the effects of space-charge forces on the beam. The use of strong transverse focusing reduces transverse emittance growth. Large transitions are avoided in the design, which provide for a wellmatched beam to reduce halo formation from mismatch.

Beam losses in the APT accelerator must be extremely low $(0.1-1.0 \mathrm{nA} / \mathrm{m})$ so as to minimize structure activation and allow hands-on maintenance. In order to meet this design criterion we have required large apertures, both transverse and longitudinal, in order to contain all the beam. The transverse apertures in the accelerator have been chosen to provide a large aperture radius to rms beam size ratio. This ratio has been used as a figure of merit for our designs. The additional

*Engineering Sciences and Applications Division 
requirement to have reasonable shunt-impedance value in order to reduce if structure-power loss, was also considered when making the choices of transverse apertures. The synchronous phase profile, which was chosen for the accelerator, allows adequate longitudinal acceptance of the beam throughout all phases of acceleration with sufficient margin to avoid the nonlinear region of the acceptance. Precision beam diagnostics and if system control will be required to monitor and correct deviations from nominal operation.

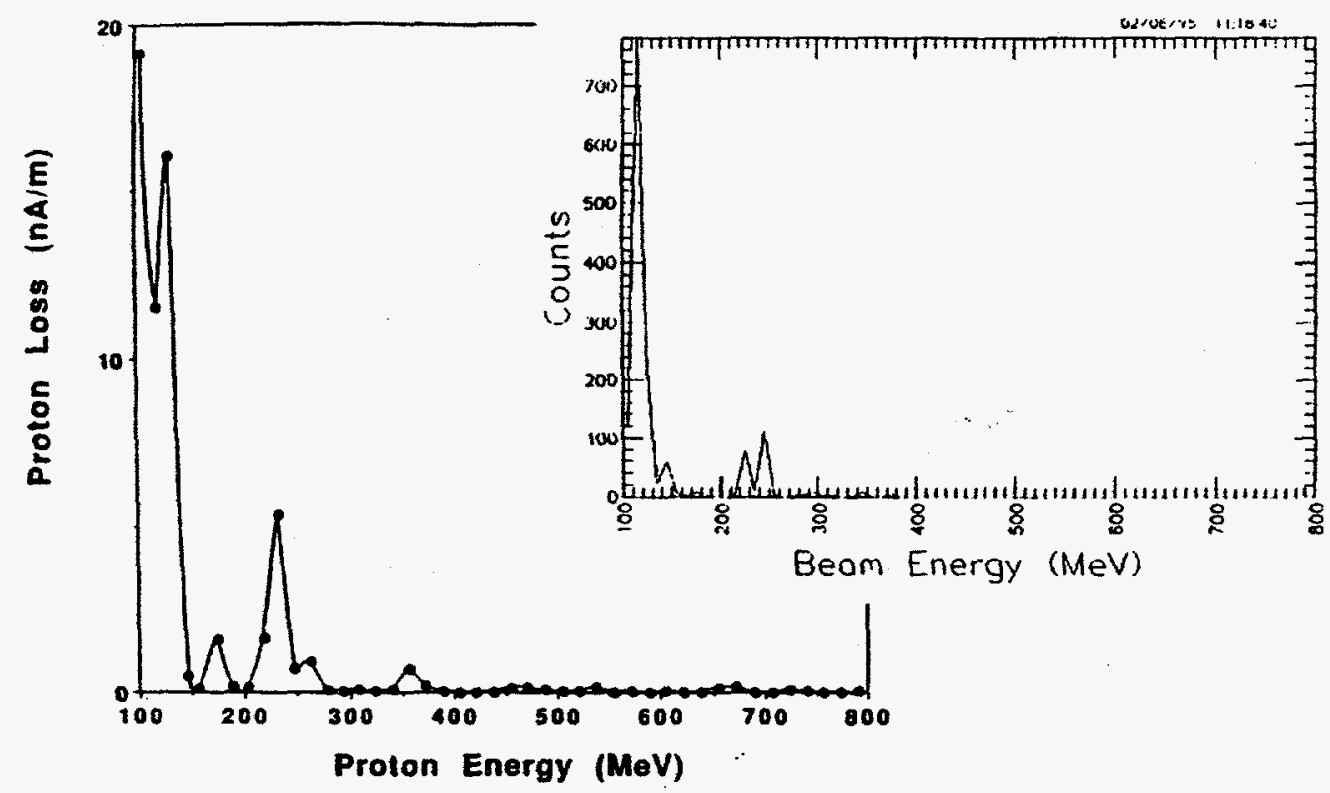

FIGURE 1. Beam loss in the LANSCE side-coupled linac from activation measurements as a function of beam energy. The inset in the upper right-hand corner is the simulation result. Measured losses are $0.1 \%$ and simulated losses are $1.2 \%$.

\section{LAMPF SIMULATIONS AND OPERATION}

Comparisons have been made between simulations and beam measurements of the LANSCE accelerator (formerly LAMPF) which have allowed us to benchmark the simulation codes [2]. The $\mathrm{rms}$ beam properties, predicted by the simulation codes, agree well with measurements. However, to accurately predict the magnitude of the beam losses, a detailed knowledge of the tails of the beam distribution is necessary. Figure 1 shows both simulation results and results from activation measurements for the LANSCE side-coupled linac (SCL) from 100 to $800 \mathrm{MeV}$. As can be seen, the locations of large beam loss are well predicted by the codes, but, the fraction of total beam lost is overpredicted by approximately an order of magnitude. The simulations indicate that the primary cause of beam loss is due to poor longitudinal capture and poor longitudinal matching. No 
longitudinal matching is done to compensate for the acceptance change due to the $201.25 \mathrm{MHz}$ to $805 \mathrm{MHz}$ frequency transition from the drift-tube linac to the SCL. Inefficient beam bunching at injection leads to low-energy losses, which is observed at LANSCE. Bunching of the beam by a radiofrequency quadrupole (RFQ) is far superior to a conventional multi-buncher system. This has been demonstrated in simulation studies for possible upgrades to LANSCE and is shown in Fig.2. The longitudinal beam distribution at $100 \mathrm{MeV}$ is seen to have an extremely long tail due to poor bunching prior to injection into the drift-tube linac at $750 \mathrm{keV}$. The strong longitudinal focusing and bunching of a RFQ can eliminate this tail as is seen in the figure. Simulations using both the RFQ beam and longitudinal matching at $100 \mathrm{MeV}$ resulted in approximately two orders of magnitude reduction in beam losses. We believe that the RFQ is a critical, required component, if a high-quality, low-loss beam is to be produced in highcurrent applications. Additionally, all of these results indicate the importance of examining the effects of longitudinal beam dynamics, including bunching and matching, to avoid beam halo and beam losses.

PRESENT

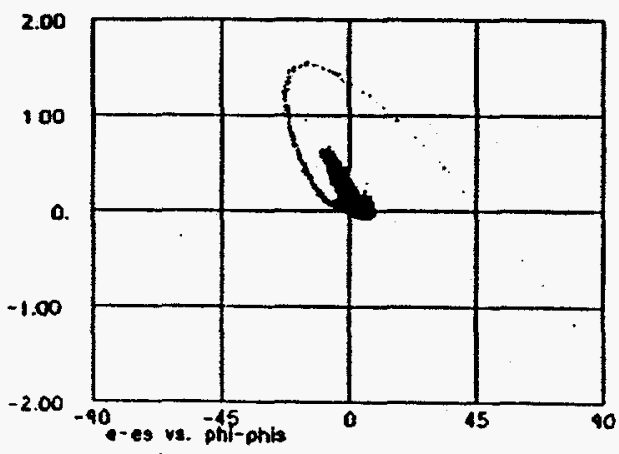

RFQ INJECTOR

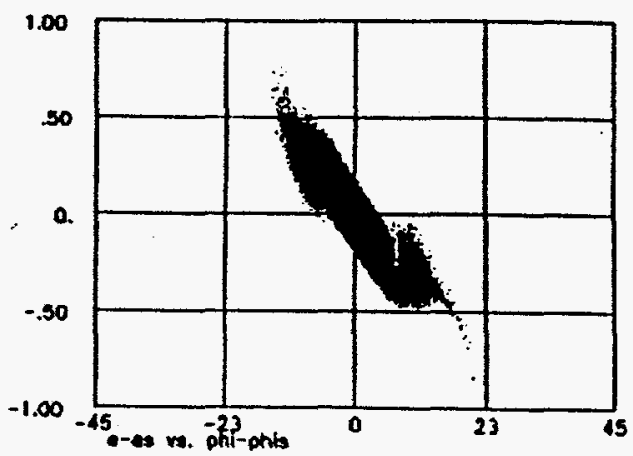

FIGURE 2. A comparison of the longitudinal phase space distributions at $100 \mathrm{MeV}$ from simulations for the present LANSCE accelerator and a modified LANSCE using a 5.4 MeV RFQ as an injector.

\section{ROOM-TEMPERATURE BASELINE DESIGN}

A schematic diagram of the room-temperature baseline design is shown in Fig. 3. Table 1 lists some parameters for the baseline design. A $100 \mathrm{~mA}$, $\mathrm{cw}, \mathrm{H}^{+}$beam is injected at $75 \mathrm{keV}$ into a $350-\mathrm{MHz} \mathrm{RFQ}$, which accelerates the beam to 6.7 $\mathrm{MeV}$ [3]. An engineering drawing of this high-energy RFQ is shown in Fig. 4. The RFQ consists of four resonantly-coupled 2-meter segments with each segment made up of two 1-meter sections. Each section is fabricated from four vane-cavity quadrants which are brazed together. The $6.7-\mathrm{MeV}$ RFQ output energy was 
chosen to provide a transition to the coupled-cavity drift-tube linac (CCDTL) structure [4] without requiring focusing quadrupoles in the drift-tubes.

The CCDTL structure, operating at a frequency of $700 \mathrm{MHz}$, will be used to accelerate the beam to $100 \mathrm{MeV}$. A generic 3-gap CCDTL structure is shown schematically in Fig. 5. The CCDTL structure is a hybrid structure combining the features of a conventional drift-tube linac and that of a coupled-cavity linac, such as the SCL. The CCDTL provides the field stability of a $\pi / 2$-mode periodic structure with the high transit-time factor required to efficiently accelerate low- $\beta$ ion beams. One of the main advantages of the CCDTL compared with the conventional DTL is that all quadrupole-focusing lenses are exterrial to the accelerating cavities rather than installed within drift tubes. The CCDTL provides good efficiency for large bore radii in a high-frequency structure, and assures better mechanical stability for the quadrupoles. In addition, the drift-tube alignment requirements are relaxed, and accurate magnet alignment is achievable. Three-gap CCDTL cavities will be used to accelerate the beam to $20 \mathrm{MeV}$. From 20 to $100 \mathrm{MeV}$, 2-gap cavities will be used to maintain the high average shuntimpedance of $\geq 25 \mathrm{M} \Omega / \mathrm{m}$. At $100 \mathrm{MeV}$, a transition is made to a conventional, $700-\mathrm{MHz} \mathrm{SCL}$, which accelerates the beam to the final energy of $1 \mathrm{GeV}$. SCL tanks with 6 cells/tank will be used from 100 to $154 \mathrm{MeV}$. Tanks having 7 cells/tank are used thereafter. The choices to use CCDTL and SCL structures allow the majority of the accelerator to operate at $700 \mathrm{MHz}$ and in the $\pi / 2$ structure mode. Because the RFQ operates at $350 \mathrm{MHz}$, only every other longitudinal bucket in the CCDTL and SCL, which both operate at $700 \mathrm{MHz}$, is filled. Therefore, a possible upgrade path, which would double the output beam current by filling all buckets at the same peak current per bunch, could be to funnel [5] another beam at some energy above $6.7 \mathrm{MeV}$.

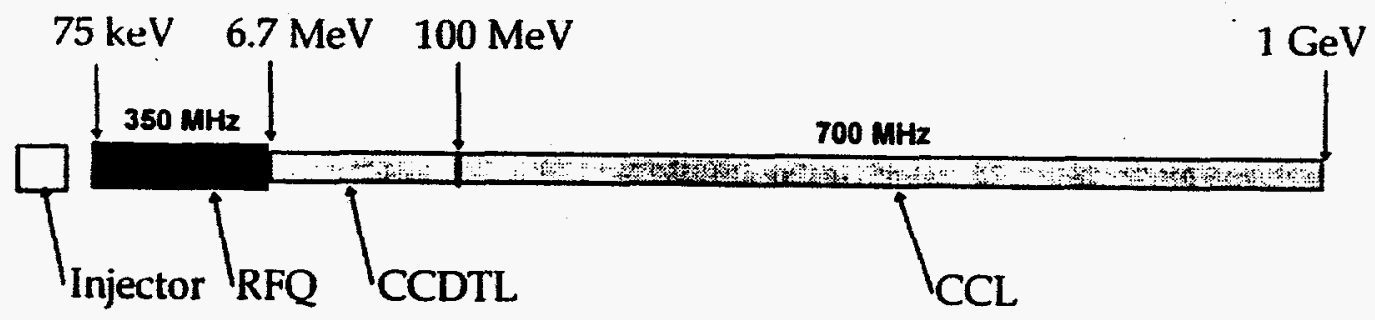

FIGURE 3. Schematic view of the proposed accelerator for tritium production.

Although transitions from one type of accelerating structure to another exist, there are no abrupt changes in the focusing period, and the beam experiences no change in the average transverse and longitudinal focusing forces at these locations. This is achieved by maintaining constant focusing through the transitions and allowing only very adiabatic changes throughout the linac. To do so, a FODO magnetic-quadrupole-focusing lattice with an $8-\beta \lambda$ period, and magnets of constant gradient times length are used from $6.7-1000 \mathrm{MeV}$. At the 
$\mathrm{RFQ} / \mathrm{CCDTL}$ transition, the RFQ transverse zero-current phase advance per unit length $\left(\sigma_{0} / \mathrm{L}\right)$ was chosen to be $1.96 \% \mathrm{~cm}$. The CCDTL transverse-focusing-lattice parameters were chosen to give the same $\sigma_{0} / \mathrm{L}$. The if phases and amplitudes in the cavities were chosen to provide adequate longitudinal focusing, and efficient acceleration. It has been found in previous studies [6], that by maintaining

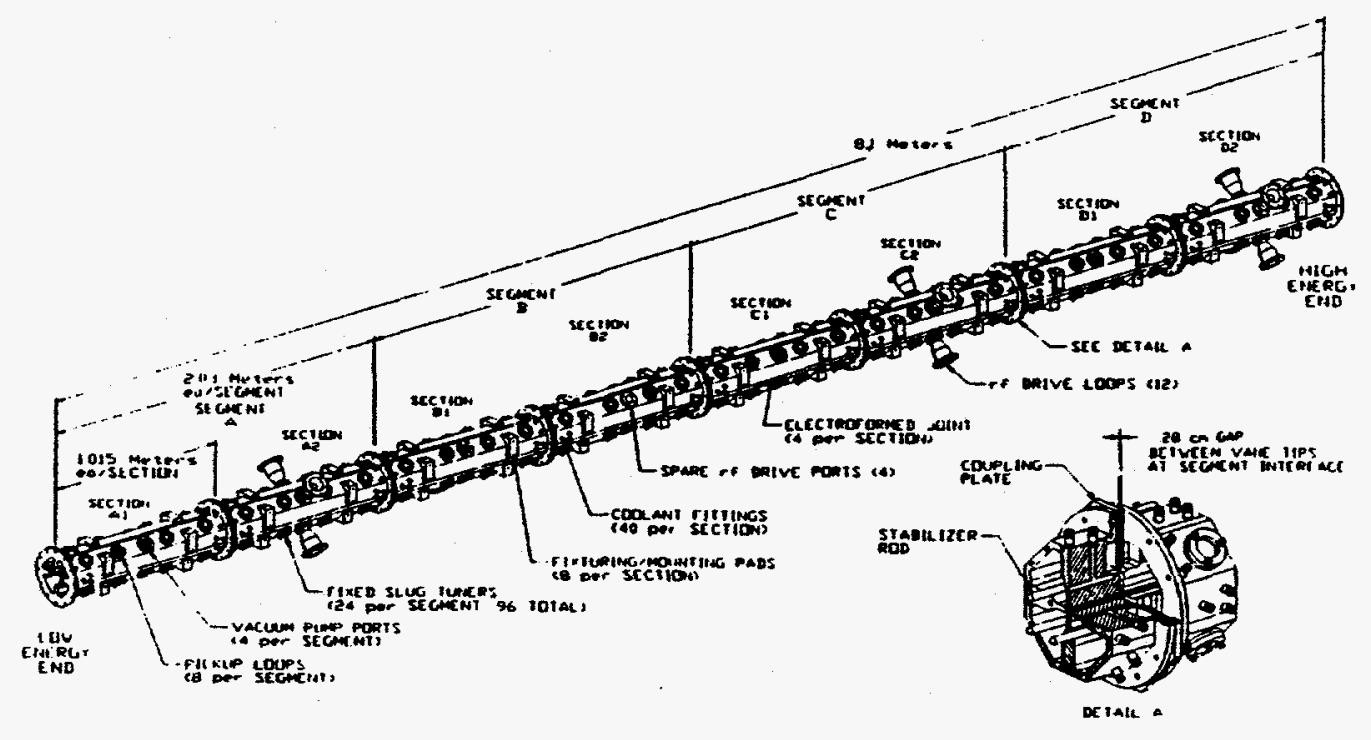

FIGURE 4. Engineering drawing of the 6.7-MeV RFQ.

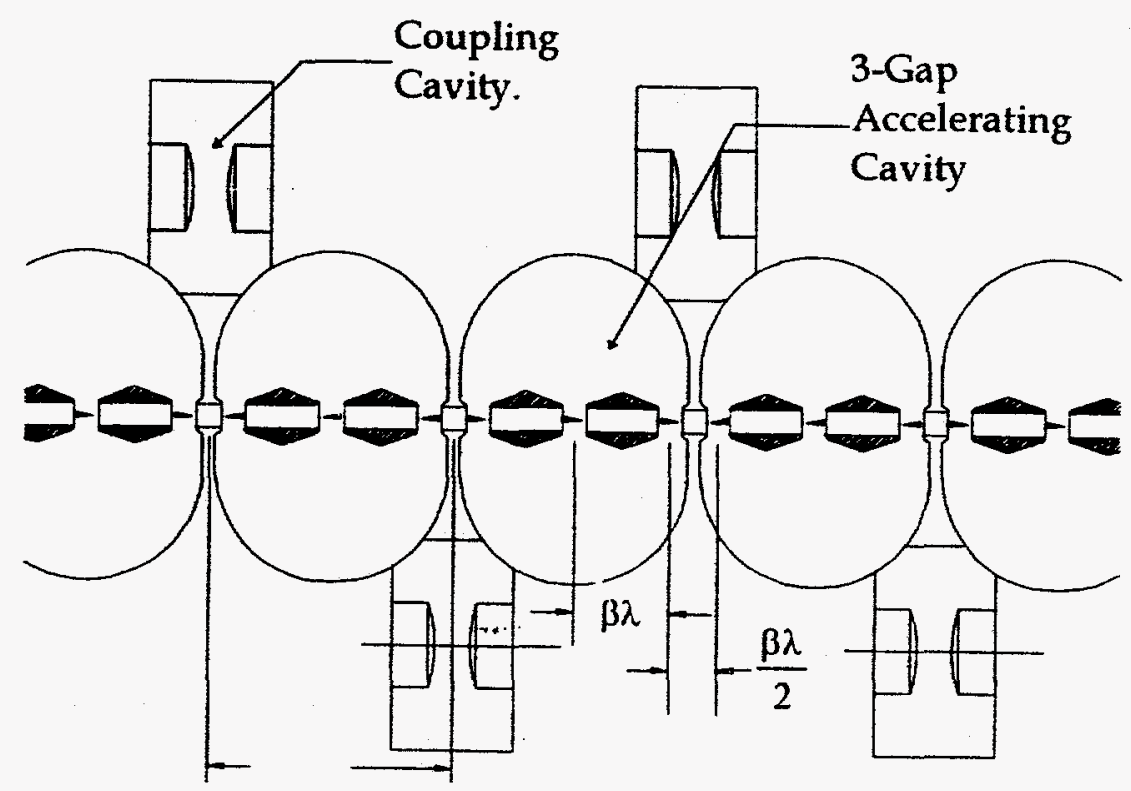

FIGURE 5. Schematic diagram of a generic 3-gap CCDTL. 
constant focusing across transitions, it is possible to design an accelerator, which is relatively insensitive to beam current. The RFQ/CCDTL transition region has been designed so that no separate matching section is required while preserving the current-independence. This has been verified using multiparticle beam-dynamics simulations.

Table 1. Baseline Room-Temperature Accelerator Parameters.

\begin{tabular}{|c|c|c|c|c|}
\hline Parameter & $\mathbf{R F Q}$ & $\begin{array}{l}\text { CCDTL } \\
\text { 3-Gap }\end{array}$ & $\begin{array}{l}\text { CCDTL } \\
\text { 2-Gap }\end{array}$ & $\begin{array}{l}\text { CCL } \\
\text { 6-cell, } \\
7 \text {-cell }\end{array}$ \\
\hline Energy (MeV) & $0.075-6.7$ & $6.7-20$ & $20-100$ & $100-1000$ \\
\hline Frequency $(\mathrm{MHz})$ & 350 & 700 & 700 & 700 \\
\hline Beam Current $(\mathrm{mA})$ & 100 & 100 & 100 & 100 \\
\hline Aperture Radius (cm) & $0.23-0.34$ & $0.75-1.0$ & $1.25-1.75$ & $1.75-2.5$ \\
\hline$E_{0} T(M V / m)$ & 1.38 & $0.85-1.87$ & $1.50-1.32$ & $1.35-1.71$ \\
\hline Real-Estate $E_{0} T(M V / m)$ & 0.81 & $0.53-1.17$ & $1.17-1.0$ & $1.0-1.5$ \\
\hline Synchronous Phase (deg) & -90 to -60 & -60 to -40 & -40 to -35 & -35 to -30 \\
\hline Shunt Impedance $(\mathrm{M} \Omega / \mathrm{m})$ & - & $30-38$ & $30-26$ & $26-41$ \\
\hline $\begin{array}{l}\text { Transverse } \\
\text { Phase Advance/Period (deg) }\end{array}$ & 20 & 80 & 80 & $80-40$ \\
\hline Quadrupole Lattice & FODO & FODO & FODO & FODO \\
\hline Quadrupole Length $(\mathrm{cm})$ & - & 3.0 & 3.0 & 3.0 \\
\hline Quadrupole Gradient (T/m) & & 87.5 & 87.5 & 87.5 \\
\hline $\begin{array}{l}\text { Transverse Emittance } \\
\text { (cm-mrad) }\end{array}$ & 0.022 & $0.022-0.024$ & 0.024 & 0.024 \\
\hline $\begin{array}{l}\text { Longitudinal Emittance* } \\
\text { (cm-mrad) }\end{array}$ & 0.044 & 0.042 & 0.048 & 0.068 \\
\hline $\begin{array}{l}\text { Ratio of Aperture Radius } \\
\text { to RMS Beam Size }\end{array}$ & - & $5-6$ & $6-13$ & $13-25$ \\
\hline
\end{tabular}

*Emittances are $\mathrm{ms}$, normalized, area/ $\pi$ values.

To guarantee high availability and reliability of the accelerator beyond the $\mathrm{RFQ}$, rf modules will consist of seven 1-MW klystrons powering approximately 200 accelerating cells. Structure studies indicate that modules containing 200 accelerating cells or less should have very stable field distributions. In this scheme, 
the accelerating structure itself acts as a manifold to accept if power from multiple drives. Therefore, the structure locks the phase and amplitude of the if field, which assures the proper longitudinal dynamics. This simplifies beam tuning and if control. Such a module requires only 6 klystrons to provide adequate power, however, fault analyses have shown that by adding a seventh redundant klystron, the If module reliability is significantly increased. All seven klystrons would be operated at lower power to increase tube lifetime. In the event of a single klystron failure, all klystrons would be brought up to high-power operation.

Figure 6 shows the transverse and longitudinal tune-depression ratios $\left(\sigma / \sigma_{0}\right.$, where $\sigma$ is the phase advance per period at full beam current) as a function of proton beam energy. As can be seen, although the average beam current is quite high (100 mA), the linac parameters have been chosen to avoid low depression ratios, which can lead to beam halo production when there is beam mismatch [7]. Figure 7 shows the equipartition ratio as a function of proton beam energy, defined as the ratio of $\sigma \varepsilon$ values for the transverse to longitudinal planes, where $\varepsilon$ is the rms normalized emittance. In the first $20 \mathrm{MeV}$ of the accelerator, where it is economical to do so, equipartitioning is maintained on average. As a result, there is very little transverse or longitudinal emittance growth up to $20 \mathrm{MeV}$ as can be

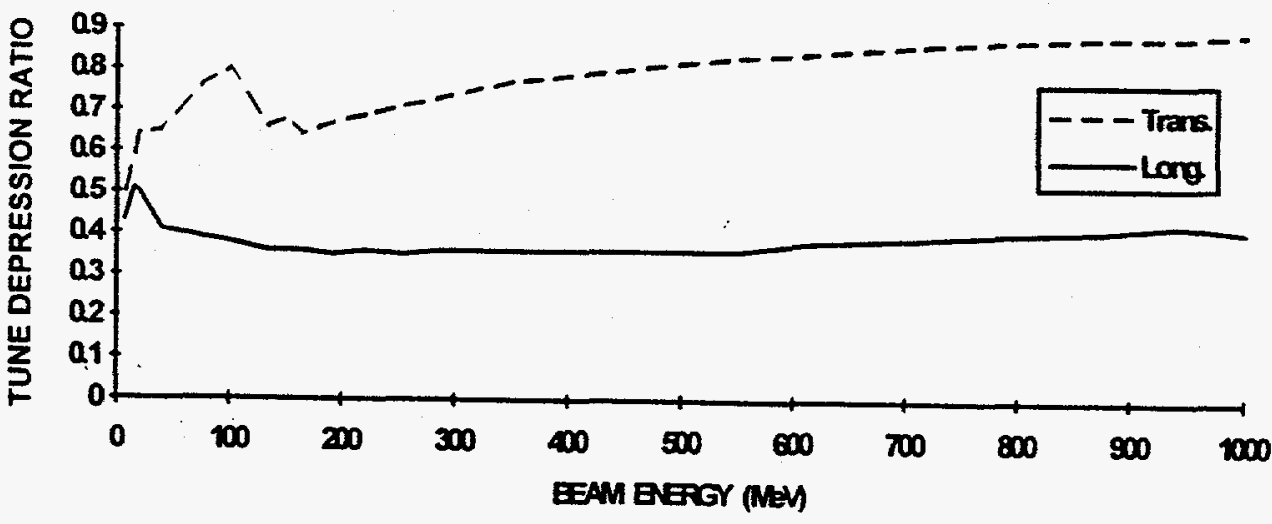

FIGURE 6. Tune depression ratios as a function of proton beam energy.

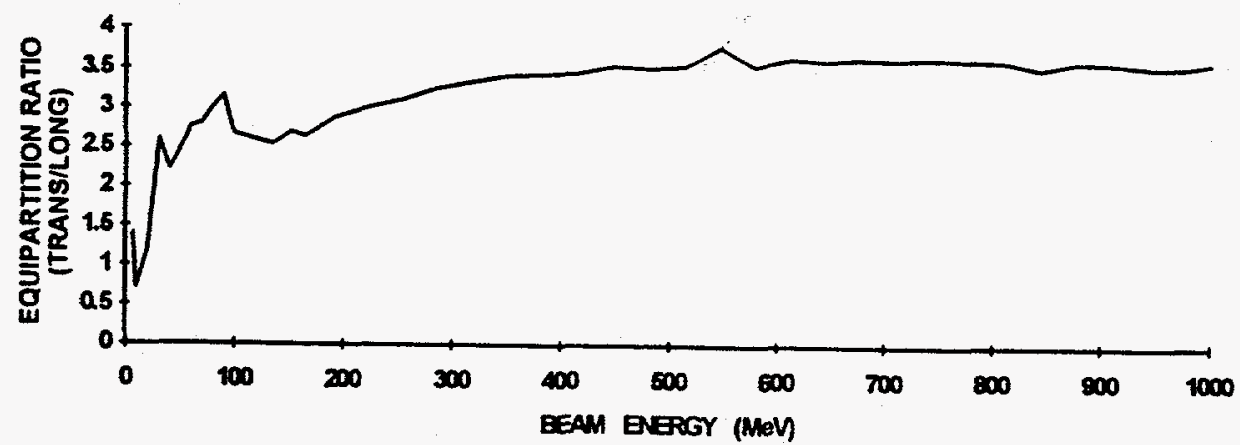

FIGURE 7. Equipartition ratio as a function proton beam energy. 
seen in Fig. 8. Strong transverse focusing allows the trans se emittance to be held constant throughout the accelerator up to $1 \mathrm{GeV}$. Because the real estate gradient in the accelerator is nearly constant at $1.5 \mathrm{MV} / \mathrm{m}$ above $100 \mathrm{MeV}$, the longitudinal focusing grows weaker as a function of beam energy. As a consequence, the longitudinal emittance is seen to grow. Because there are no specific beam brightness or luminosity requirements for this application, this longitudinal-emittance growth is tolerable, as long as it does not affect the beam loss along the machine. The simulation results indicate that a large transverse aperture radius to $\mathrm{ms}$ beam-size ratio is maintained throughout the high-energy section of the accelerator, where structure activation from beam loss is of the greatest concern. The values of this ratio are also included in Table 1 . Our studies show that reducing the transverse focusing strength above $100 \mathrm{MeV}$ to bring the equipartition ratio closer to unity, as proposed elsewhere [8], has little effect on the emittance growths, but increases the transverse rms beam size, which increases the risk of beam loss.

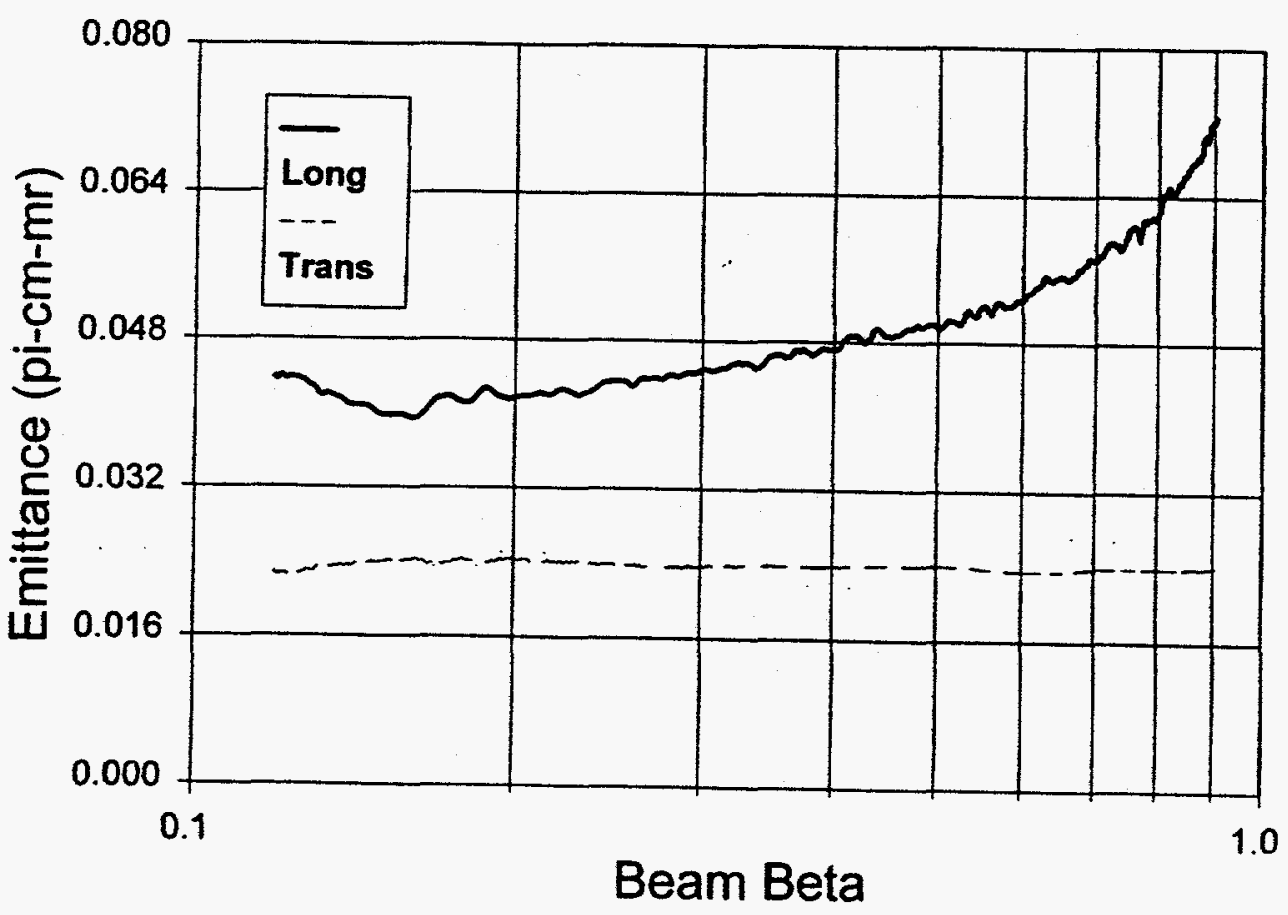

FIGURE 8. Normalized rms emittances as a function of beam velocity, $\beta$. 


\section{HIGH-ENERGY SUPERCONDUCTING OPTION}

Recently, we have examined the feasibility of using superconducting rf cavities to accelerate the proton beam in the high-energy section of the linac from 100 to $1000 \mathrm{MeV}$. Cost studies indicate that as much as twenty million dollars per year could be saved in operating costs by using this technology. However, this technology is viewed by some as high risk. The purpose of our study was to develop a point design to a sufficient level so that technical feasibility, technology development requirements, risk factors, and cost could be determined.

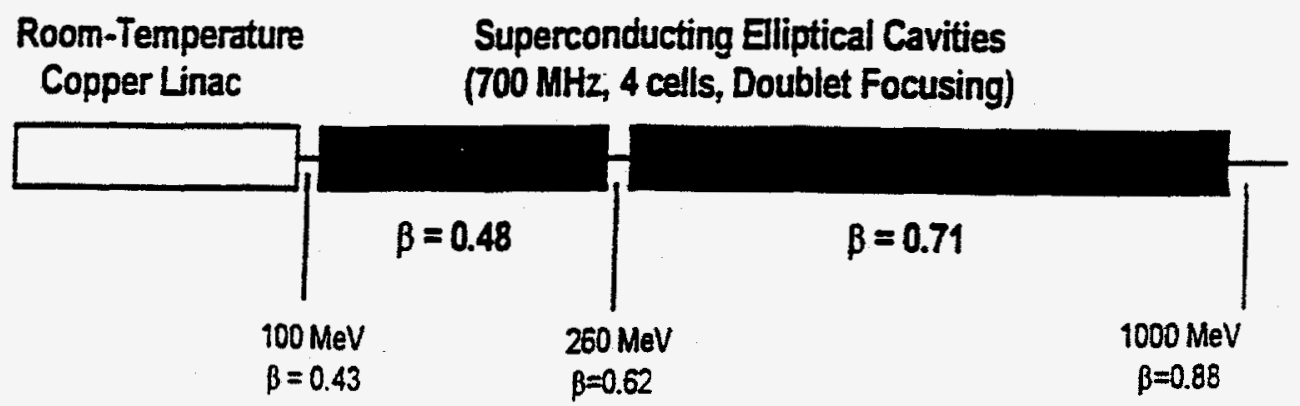

FIGURE 9. Schematic view of the high-energy superconducting option.

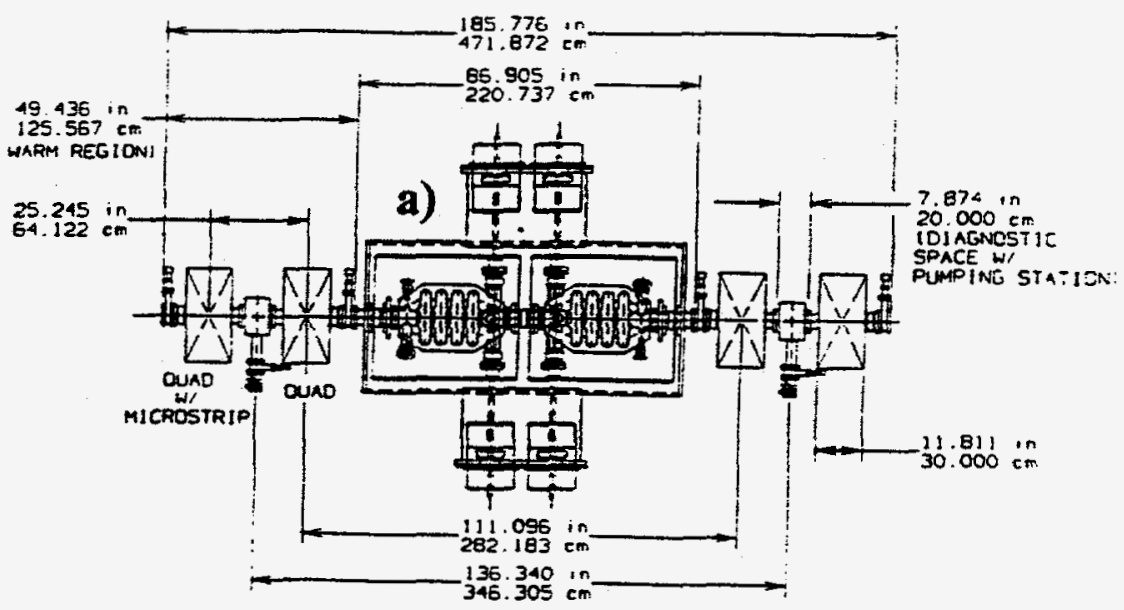

FIGURE 10. Engineering layout of a medium- $\beta$ cryomodule. The distance from the midpoint between a pair of quadrupoles to the next midpoint is equal to one FDO period. 
The superconducting if linac (SCRFL) presented here is unoptimized. We have chosen conservative requirements for the various system components, most of which have already been demonstrated in existing accelerators or laboratory tests.

Figure 9 shows a schematic diagram of the proposed SCRFL, and Table 2 gives some of the accelerator parameters. The SCRFL consists of two sections called the medium- $\beta$ and high- $\beta$ sections. Each section is composed of identical 4cell elliptical cavities, where each cell length is $\beta \lambda / 2$. The medium- $\beta$ section has cavities that are optimized at $\beta=0.48$ and will accelerate the beam from 100 to 261 $\mathrm{MeV}$. The cavities in the high- $\beta$ section are optimized at $\beta=0.71$, and will accelerate the beam up to $1000 \mathrm{MeV}$. A cryostat containing two cavities forms a cryomodule. Figure 10 shows an engineering drawing of a medium- $\beta$ cryomodule, and one period of the magnetic quadrupole focusing lattice. In this example, transverse focusing is provided by quadrupole doublets between each cryomodule. The power from each klystron will be split among four cavities. RF power will be fed to each cavity using two antenna-type power couplers capable of handling 105 $\mathrm{kW}$, each.

The use of short, multi-cell if cavities has the advantage that a relatively high transit-time factor can be maintained, while having a large velocity acceptance. Figure 11 shows the transit-time factor for various multi-cell cavities as a function of the ratio of beam $\beta$ to cavity design $\beta$. We have chosen 4-cell cavities in this example as a compromise to minimize the number of required system components, while maintaining a large velocity acceptance.

Table 2. High-Energy Superconducting Accelerator Parameters.

\begin{tabular}{ll} 
Parameter & \\
\hline Energy Range (MeV) & $100-1000$ \\
Frequency (MHz) & 700 \\
Beam Current (mA) & 100 \\
No. of $\beta$ Sections & 2 \\
No. of Cavities & 488 \\
No. of Cryostats & 244 \\
No. of Klystrons & 122 \\
Cavities/Cryostat & 2 \\
Cavities/Klystron & 4 \\
Cells/Cavity & 4 \\
RF Couplers/Cavity & 2 \\
RF Power/Klystron (MW) & 0.67 (med. $\beta$ ), 1.0 (high $\beta)$ \\
RF Power/Coupler (kW) & 72 (med. $\beta$ ), 105 (high $\beta)$ \\
Accelerating Field, $E_{\mathrm{a}}(\mathrm{MV} / \mathrm{m})$ & $4.2-5.3$ \\
Average Phase (deg) & -35 \\
Aperture Radius (cm) & 5.0 (med. $\beta$ ) and 7.2 (high $\beta$ ) \\
\hline
\end{tabular}

The large velocity acceptance of these cavities allows operational flexibility. In normal operation, the multi-cell cavities will be operated for a specific energy 
gain per cavity (medium $\beta \Delta \mathrm{W}=1.44 \mathrm{MeV}$, high $\beta \Delta \mathrm{W}=2.1 \mathrm{MeV}$ ) with an average synchronous phase of $-35^{\circ}$. An iterative procedure, done with a computer, is used to determine the required if field amplitude and injection phase for each cavity, such that the desired energy gain per cavity and average synchronous phase is achieved. Therefore, the cavity if amplitudes vary as a function of beam energy. Simulation results show emittance growths between 100 to $1000 \mathrm{MeV}$ of $25 \%$ and $8 \%$, respectively, for the transverse and longitudinal degrees of freedom. The ratio of transverse aperture radius to $\mathrm{rms}$ beam size in the superconducting sections ranges from 19 to 26 .

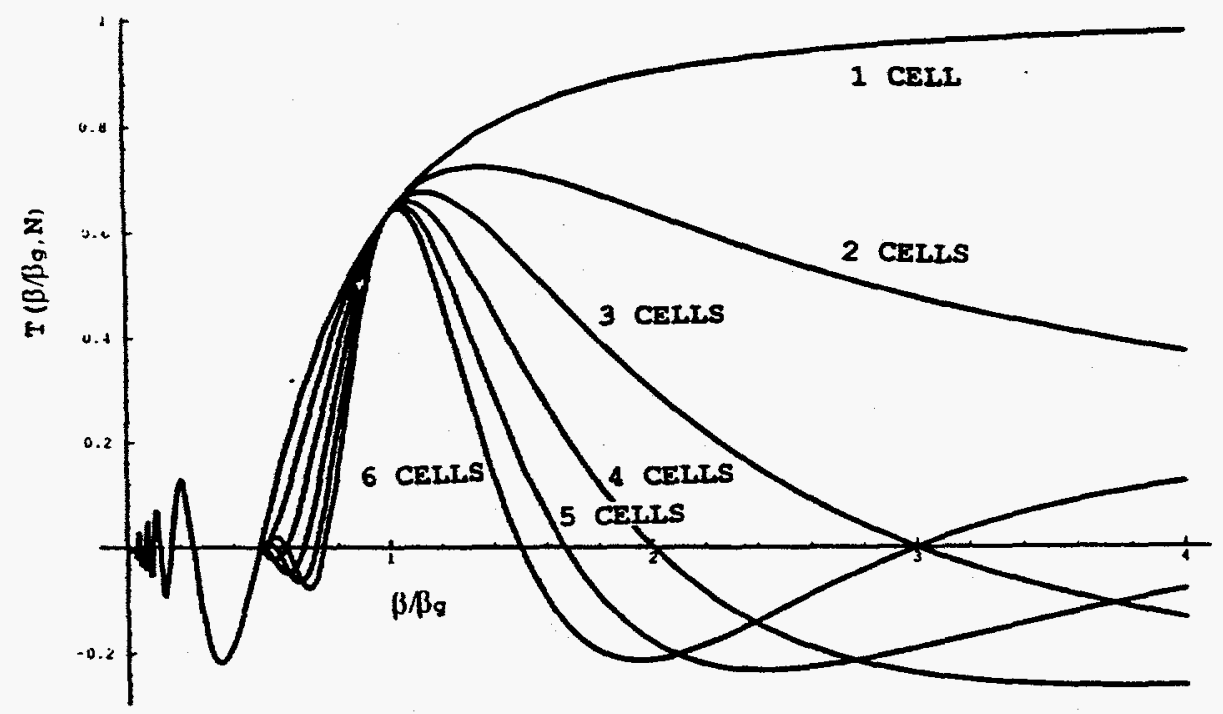

FIGURE 11. Transit-time factor for various multi-cell cavities as a function of the ratio of beam $\beta$ to cavity design $\beta$.

To investigate alternative operating schemes that use the inherent flexibility of a linac built from independently-phased resonators, three examples were simulated. The simulation results are given in Table 3, below. Scheme 1 assumes that all cavities will be operated at a constant accelerating field of $E_{a}=5.3 \mathrm{MV} / \mathrm{m}$. This is the maximum field under normal operating conditions. In this scheme, the energy gain per cavity is no longer fixed. We have assumed a cavity average synchronous phase of $-35^{\circ}$. As can be seen, the beam output energy is raised by $99 \mathrm{MeV}$. The change in output beam emittances and ratio of transverse aperture to $\mathrm{rms}$ beam size is small. Also shown in Table 3 is the minimum required beam current to produce $100 \mathrm{MW}$ output beam power at $1099 \mathrm{MeV}$. This example demonstrates an alternative operating scheme which could be used in the event of source output current degradation. In Scheme 2, the average synchronous phase has been reduced to $-25^{\circ}$. As is expected, the output energy is further increased to 1179 $\mathrm{MeV}$. In Scheme 3, the cavity fields have been increased by $33 \%$. This scheme demonstrates a possible upgrade path, which would require significantly increased 
power-coupler capabilities and klystron output to produce $130 \mathrm{MW}$ of beam power, without requiring additional accelerating cavities. In the last two schemes, there is a slight degradation in the ratio of transverse aperture to rms beam size. Transverse emittance growth is observed in all cases, which is comparable to the $25 \%$ observed for the nominal operating mode. The effects of emittance growth on beam uniformity at the neutron production target have not been studied.

Table 3. Alternative operating schemes for the high-energy superconducting option. Required beam current is the beam current required to produce a $100-\mathrm{MW}$ beam power.

\begin{tabular}{lllllll}
\hline Case & $\begin{array}{l}\text { Output } \\
\text { Energy } \\
\text { (MeV) }\end{array}$ & $\begin{array}{l}\text { Trans. } \\
\text { Emittance } \\
\text { Growth }\end{array}$ & $\begin{array}{l}\text { Long. } \\
\text { Emittance } \\
\text { Growth }\end{array}$ & $\begin{array}{l}\text { Required } \\
\text { Beam } \\
\text { Current }\end{array}$ & $\begin{array}{l}\text { Aperture } \\
\text { Ratio, } \\
\text { Med. } \beta\end{array}$ & $\begin{array}{l}\text { Aperture } \\
\text { Ratio, } \\
\text { High- } \beta\end{array}$ \\
\hline 1 & 1099 & $17 \%$ & $-5 \%$ & $91 \mathrm{~mA}$ & 18 & 21 \\
2 & 1179 & $32 \%$ & $98 \%$ & $85 \mathrm{~mA}$ & 18 & 20 \\
3 & 1297 & $19 \%$ & $-4 \%$ & $77 \mathrm{~mA}$ & 17 & 20 \\
\hline
\end{tabular}

Experience at operating superconducting accelerator facilities has shown that often there is a large variation in the maximum accelerating gradients achieved in identical multi-cell accelerating cavities. Typically these are $\beta=1$ cavities used to accelerate electron beams such as at CEBAF [9]. If cavities fail or perform at lower than expected accelerating gradients, the gradients and if phases in the other cavities are adjusted to compensate and provide the required additional energy gain. A possible solution to increase the availability of the proposed superconducting linac is to provide additional accelerating cavities, thus anticipating some fraction of cavity failures. In order to examine these effects, we simulated the case where $5 \%$ of all cavities are not operating (every 20th cavity off). To restore the beam energy, we added $5 \%$ additional cavities to the high- $\beta$ section. Simulation results, using a simple algorithm for setting the cavity phases, showed a transmission of $100 \%$ with a reduced output beam energy of $993.4 \mathrm{MeV}$ for this case. Small adjustments of the phases should restore the correct final beam energy. The transverse and longitudinal emittances were observed to grow by factors of 2.9 and 6.8 , respectively; however, only small changes were observed in the aperture to rms values.

Additional simulation studies of various error, alignment, and failure conditions have also been completed for this point design. These studies indicate that the alignment tolerances required for the system proposed are greatly relaxed compared to the room-temperature design. Relatively large if phase and if amplitude errors are tolerable $\left(5^{\circ}\right.$ and $3 \%$ ). A maximum of two consecutive quadrupole doublets can fail without beam loss although there is a substantial reduction in the ratio of transverse aperture to rms beam size. Our initial studies indicate that the superconducting linac is tolerable to many imperfections and fault conditions. 


\section{SUMMARY}

The details of our baseline design for a room-temperature linear accelerator for tritium production have been discussed. The main new features of this design are the use of a high-energy RFQ to accelerate the beam to $6.7 \mathrm{MeV}$ and a new medium- $\beta$ structure from 6.7 to $100 \mathrm{MeV}$, the CCDTL. The design presented here is conservative and will meet the design requirement for low beam loss and hands-on maintenance.

We have also discussed the option of using superconducting cavities in the high-energy section of the accelerator from 100 to $1000 \mathrm{MeV}$. The two main advantages of the superconducting option may be increased operational flexibility and substantial power savings. Table 4 shows a comparison between the performance of the proposed room-temperature, SCL and the superconducting option. Although the beam evolution in the two linacs is quite different, both will meet the requirements for accelerator production of tritium.

Table 4. Simulation results. A comparison of the performance of the room-temperature baseline design and the superconducting option for $100-1000 \mathrm{MeV}$.

\begin{tabular}{lll}
\hline & SCRF Linac & $\begin{array}{l}\text { Room-Temperature } \\
\text { Baseline }\end{array}$ \\
\hline $\begin{array}{l}\text { Transverse } \\
\text { Emittance Growth }\end{array}$ & $25 \%$ & None \\
$\begin{array}{l}\text { Longitudinal } \\
\text { Emittance Growth }\end{array}$ & $8 \%$ & $41 \%$ \\
Transverse & & \\
Aperture To RMS Ratio & $19(100 \mathrm{MeV})$ & $13(100 \mathrm{MeV})$ \\
& $24(260 \mathrm{MeV})$ & $21(260 \mathrm{MeV})$ \\
Transverse Tune Depression Ratio & $0.50(100 \mathrm{MeV})$ & $0.80(1000 \mathrm{MeV})$ \\
$\sigma_{t} / \sigma_{\text {ot }}$ & $0.81(260 \mathrm{MeV})$ & $0.71(260 \mathrm{MeV})$ \\
& $0.98(1000 \mathrm{MeV})$ & $0.89(1000 \mathrm{MeV})$ \\
Longitudinal Tune Depression Ratio & $0.53(100 \mathrm{MeV})$ & $0.38(100 \mathrm{MeV})$ \\
$\sigma_{l} / \sigma_{\text {ol }}$ & $0.71(260 \mathrm{MeV})$ & $0.35(260 \mathrm{MeV})$ \\
& $0.94(1000 \mathrm{MeV})$ & $0.42(1000 \mathrm{MeV})$ \\
\hline
\end{tabular}




\title{
REFERENCES
}

1. T. P. Wangler et al., "Linear Accelerator for Production of Tritium: Physics Design Challenges," Proceedings of the 1990 Linear Accelerator Conference, Albuquerque, New Mexico, September 10-14, 1990.

2. R. W. Garnett, E. R. Gray, L. J. Rybarcyk, and T. P. Wangler, "Simulation Studies of the LAMPF Proton Linac," Proceedings of the 1995 Particle Accelerator Conference and International Conference on High-Energy Accelerators, Dallas, Texas, May 1-5, 1995.

3. L. M. Young, "An 8-Meter-Long Coupled Cavity RFQ Linac," Proceedings of the 17th International Linac Conference, Tsukuba, Ibaraki, Japan, August 21-26, 1994.

4. J. H. Billen, "A New Structure for Intermediate-Velocity Particles," Proceedings of the 17th International Linac Conference, Tsukuba, Ibaraki, Japan, August 21-26, 1994.

5. S. Nath, "Funneling in LANL High-Intensity Linac Designs," Proceedings of the Accelerator-Driven Transmutation Technologies Conference, Las Vegas, Nevada, July 25-29, 1994.

6. R. S. Mills, K. R. Crandall, and J. A. Farrell, "Designing Self-Matching Linacs," Proceedings of the 1984 Linear Accelerator Conference, Seeheim, Germany, May 7-11, 1984.

R. W. Garnett and P. Smith, "Design of a Current-Independent Matching Section for APDF," Proceedings of the 17th International Linear Accelerator Conference, Tsukuba, Ibaraki,Japan, August 21-26, 1994.

7. J. S. O'Connell, T. P. Wangler, R. S. Mills, and K. R. Crandall, "Beam Halo Formation From Space-Charge Dominated Beams in Uniform Focusing Channels," Proceedings of the 1993 Particle Accelerator Conference, Washington D.C., May 17-20, 1993.

8. M. Reiser, "Beam Physics Design Strategy for a High-Current RF Linac," Proceedings of the Accelerator-Driven Transmutation Technologies Conference, Las Vegas, Nevada, July 25-29, 1994; Also see M. Reiser et al., this conference.

9. C. Reece et al., "Performance Experience with the CEBAF SRF Cavities," Proceedings of the 1995 Particle Accelerator Conference, Dallas, Texas, May 1-5, 1995. (To be published).

\section{DISCLAIMER}

\begin{abstract}
This report was prepared as an account of work sponsored by an agency of the United States Government. Neither the United States Government nor any agency thereof, nor any of their employees, makes any warranty, express or implied, or assumes any legal liability or responsibility for the accuracy, completeness, or usefulness of any information, apparatus, product, or process disclosed, or represents that its use would not infringe privately owned rights. Reference herein to any specific commercial product, process, or service by trade name, trademark, manufacturer, or otherwise does not necessarily constitute or imply its endorsement, recommendation, or favoring by the United States Government or any agency thereof. The views and opinions of authors expressed herein do not necessarily state or reflect those of the United States Government or any agency thereof.
\end{abstract}

